\title{
Approach towards Bouveret Syndrome-We Differ in Opinion
}

\author{
Mohit Bhagchandani ${ }^{1}$ (D) Ankur Sharan ${ }^{1}$
}

Received: 17 June 2020 / Accepted: 18 June 2020 / Published online: 25 June 2020

(C) Association of Surgeons of India 2020

\section{To the Editor in Chief}

Indian Journal of Surgery

We have read the article "Bouveret's Syndrome - an Unusual Fistula" by Duarte et al. published on 30 April 2020 in your esteem journal[1] with upmost interest and would like to put our point of view in the manner this patient was treated and the management protocol which was followed by the reporting doctors.

The patient was a 79 yrs woman presented with vomiting and abdominal pain, shock and signs of peritonitis with a history of hypertension as well as diabetes (glycemic status was not mentioned). The clinical condition obviously was low. The investigations revealed the condition of the patient had already deteriorated as evident from the leukocytosis and circulatory instability.

A heroic management approach was undertaken by doing distal partial gastrectomy (Billroth II) and cholecystectomy to which patient succumbed $24 \mathrm{~h}$ after surgery. The authors have not mentioned as to how much time after the patient arrived in the hospital such a major surgery was undertaken.

We were wondering if it would have been a better approach to manage this patient on expectant line of treatment by aggressive medical management, viz., decompressing the stomach contents (the CT shows stomach is full), keeping the patient nil per oral, managing the ileus with intravenous fluid, electrolyte correction, and antibiotics. Supportive treatment with oxygen, glycemic control, and attention to her other medical conditions should have been pursued with good nursing care. There may have been a possibility of requisiting a gastroenterologist on the team to dislodge the stone or extract the stone from the duodenum. A possible endo-biliary stenting may have taken care of biliary drainage (pneumobilia). In our opinion, a non-operative treatment for the first few days for this elderly lady may have been a better option. A perforated gall bladder that has involved the duodenum can at times get dried up and may not require surgical cholecystectomy. Only if pneumoperitoneum or peritonitis had increased a damage control surgery could have been planned at a later stage. A lesser surgery like peritoneal drainage and enterotomy to remove the stone would have been a better choice than cholecystectomy and partial distal gastrectomy, as published subsequently in the journal.[2, 3] We would like to hear back from the authors regarding the concerns we have raised regarding your approach in the case presented in this article.

Thank you.

\section{Compliance with Ethical Standards}

Conflict of Interest The authors declare that they have no conflicts of interest.

\section{References}

1. Duarte ML, Traple FAL, de Almeida Prado JLM (2020) Bouveret's syndrome - an unusual fistula. Ind J Surg. https://doi.org/10.1007/ s12262-020-02226-7

2. Franklin ME Jr, Dorman JP, Schuessler WW (1994) Laproscopic treatment of gallstone ileus: a case report and review of the literature. J Laproendosc Surg 4940:265-272

3. Viswanath UVA, George A, Rajan A (2020) Cholecystoduodenal fistula causing gallstone ileus. Indian J Surg. https://doi.org/10. 1007/s12262-020-02381-x

Publisher's Note Springer Nature remains neutral with regard to jurisdictional claims in published maps and institutional affiliations.

Mohit Bhagchandani 